\title{
ESTUDO DAS AUSÊNCIAS DA EQUIPE DE ENFERMAGEM
}

\author{
THE STUDY ABOUT NURSERY GROUP LACK \\ ESTUDIO DE LAS AUSENCIAS DEL EQUIPO DE ENFERMERÍA
}

Janaína Haidê Rodrigues Belem ${ }^{1}$
Raquel Rapone Gaidzinski ${ }^{2}$

RESUMO: As ausências dos elementos da equipe de enfermagem comprometem a organização do trabalho causando sobrecarga dessa equipe, e queda na qualidade da assistência de enfermagem. $\mathrm{O}$ presente estudo caracteriza o tipo e distribuição das ausências da equipe de enfermagem em um hospital geral de grande porte, propondo um Índice de Segurança Técnica para cobertura das ausências a partir da realidade investigada. Foram estudadas 8 tipos de ausências entre previstas e não previstas. O maior número de tipos de ausências encontradas foram aquelas referentes à ausências previstas, em seqüência encontramos licença maternidade representando o maior número de ausências não previstas.

PALAVRAS CHAVE: Ausência, Absenteísmo, Equipe de enfermagem

ABSTRACT: The absence of elements of nursing staff jeopardizes the work organization causing overload on this staff and reduction of quality on the nursing assistance. The present study characterizes the type and distribution of absences of the nursing staff in a general far-reaching hospital, purposing a Technical Safety Rate to cover the absences from the reality investigated. Eight types of absences were studied between foreseen or non-foreseen. The highest number of absence types found were those ones referred to the foreseen absences. In sequence we found maternity leave representing the highest number of non-foreseen absences.

KEYWORDS: Absence, Absenteeism, Nursing staff

RESUMEN: Las ausencias de los elementos del equipo de enfermería compromenten a la organización del trabajo causado sobrecarga de ese equipo y una disminución de la calidad de la asistencia de enfermería. El presente estudio caracteriza el tipo y distribución de las ausencias del equipo de enfermería en un hospital general de gran tamaño, proponiendo un Índice de Seguridad Técnica para cubrir las ausencias partir de la realidad investigada. Fueron estudiadas 8 tipos de ausencias entre previstas y no prevista. El mayor número encontrado de acuerdo al tipo de ausencias fueron aquellas referentes a las ausencias previstas, en consecuencia también encontramos licencia por maternidad representando un número mayor de ausencias no previstas.

PALABRAS CLAVE: Ausencia, Ausentismo, Equipo de enfermería

\footnotetext{
${ }^{1}$ Bolsista do $7^{\circ}$ semestre do Curso de Graduação em Enfermagem da Escola de Enfermagem USP, bolsista de Iniciação Cietífica - CNPq

${ }^{2}$ Enfermeira. Professora Doutora do Departamento de Orientação Profissional da Escola de Enfermagem da USP.
} 


\section{INTRODUÇÃO}

De acordo com a política de corte de custos imposta à área de saúde e às áreas sociais no país, encontramos um panorama de redução de pessoal de enfermagem contratado nas instituições de saúde, o que provoca novos direcionamentos no gerenciamento da equipe de enfermagem. Sabemos que para proporcionarmos um bom nível de assistência de enfermagem faz-se necessário um número adequado de funcionários. Neste cenário, as ausências dos funcionários ao trabalho contribuem para agravar as dificuldades encontradas, principalmente no tocante ao gerenciamento de enfermagem e à qualidade da assistência prestada. Por conseguinte, faz-se necessário compreender a magnitude e as causas deste problema vislumbrando estabelecer medidas de controle dessa situação.

Na literatura várias definições e abordagens têm sido apresentadas no que se refere à ausência dos funcionários ao trabalho, genericamente denominada de absenteísmo. O absenteísmo é definido por Felt (1982), Platiet al (1990), Robazzi et al (1990) como o hábito de faltar ao trabalho diário, voluntária ou involuntariamente. Chiavenato (1991) considera como a resultante da soma dos períodos em que os empregados encontra-se ausentes ao trabalho, seja por falta ou atraso, quando se esperava que estivessem presentes. Frenkiel (1980) sugere que se exclua deste conceito as ausências regidas por legislação específica e a ORGANIZACION INTERNACIONAL DEL TRABAJO (1989) recomenda a exclusão de férias e folgas do cálculo desse índice pois tratam-se de ausências previstas.

A leitura destas publicações indica que existem várias abordagens para o tema, condicionadas pela valorização de diferentes aspectos do fenômeno, o que resulta em definições diversas para um mesmo termo. Segundo Gaidzinski et al (1998) absenteísmo é o comportamento de faltar ao trabalho com freqüência, praticado por um indivíduo ou grupos de indivíduos, independente das causas que o motiva e das conseqüências que provoca. O comportamento de faltar implica em tomar a decisão de não comparecer ao trabalho, o que exclui as ausências por folgas, férias e licenças programadas.

Pretendendo dar maior clareza ao assunto, Gaidzinski et al (1993) classificaram as ausências em: ausências previstas e ausências não previstas. Dessa forma, as ausências previstas são aquelas de direito do trabalhador, como as férias e as folgas por descanso remunerado semanal e dias feriados. As ausências não previstas são consideradas as faltas abonadas, justificadas, injustificadas, as suspensões, bem como, as licenças de direito do trabalhador, uma vez que a maioria das vezes, não podem ser previsíveis como licença por doença, maternidade, paternidade, etc.

A literatura pertinente ao assunto tem mostrado que o índice de ausências não previstas varia de acordo com alguns fatores como: tipo de trabalho, grau de escolaridade, sexo, condições de trabalho, principalmente condições onde há o favorecimento do desenvolvimento de determinadas doenças. Couto (1987) classifica essas ausências em: relacionadas ao trabalho, sociais, culturais, de personalidade e por doenças.

Outros motivos relacionados às ausências são descritos no estudo de 
Rogers et al. (1990) como problemas relacionados ao transporte até o local de trabalho e fatores que influenciam a motivação do trabalhador, como por exemplo, a extensão da jornada, o estresse do campo de trabalho, relacionamento interpessoal com o grupo, o estilo de liderança do supervisor, oportunidades de promoção.

A falta de motivação do funcionário é um fator atrelado às ausências, como refere Flippo (1970) "é importante que o empregado seja capaz não apenas de trabalhar, mas tenha também boa vontade de fazê-lo. Esta boa vontade, em grande parte, apoia-se na habilidade da administração em integrar interesses e necessidades de seus empregados, com os objetivos da organização". Para Sullivan;Decker (1992), a motivação dos trabalhadores é afetada por alguns fatores, relacionados à: práticas organizacionais como por exemplo, comitês de funcionários para reivindicar melhorias; cultura de faltar; relacionamento interpessoal no ambiente de trabalho e o próprio trabalho (estresse no ambiente e relacionado ao próprio trabalho, o estilo de liderança da chefia, oportunidades de promoção, etc.); e características pessoais, como idade, nível educacional, responsabilidades familiares e quaisquer outros fatores que afetar as atitudes dos funcionários.

A equipe de enfermagem é composta, basicamente por mulheres. Assim, precisamos considerar a inserção da mulher na sociedade e no mercado de trabalho, para entender quais são os fatores externos que as levam a ausentar-se.

Steagel-Gomes; Mendes (1995) ressaltam que a mulher hoje encontra-se na situação de chefe de família ou, ainda the é incumbida a responsabilidade de complementar a renda da família. Muitas vezes, trabalha em até dois empregos além das responsabilidades que lhe competem no lar, o que pode acarretar estresse pelo excesso de trabalho, e faltas para cuidar de assuntos relacionados à família, principalmente no que concerne ao cuidado dos filhos, companheiro e pais ou parentes próximos, sendo agravado este problema quando não existe o suporte para que desenvolva as atividades domésticas como creches, escolas, moradia apropriada ou situação familiar estressante (separação do parceiro, por exemplo).

Alves (1996), também, chama a atenção para a existência de dupla jornada de trabalho dessa mulher que, além dos papéis de mãe e profissional, muitas vezes precisa manter mais de um emprego e fazer horas extras para satisfazer as necessidades de sua família.

Loman (1993) estudando as causas de absenteísmo relacionadas com os filhos de trabalhadores de enfermagem, obteve como resultado, uma grande quantidade de relatos justificando as ausências com cuidados aos filhos doentes, e em menor nível, dificuldade em conseguir outros cuidadores para os filhos.

No estudo de Dias;Avelar (1997) ocorre a agregação de fatores anátomobiológicos, fisiológicos e psicológicos como fatores internos acarretadores de faltas. Este estudo considerou como estímulos externos os agentes químicos, físicos, mecânicos, microbiológicos e outros, e como estímulos internos os pensamentos, sentimentos, expectativas, preocupações, necessidades afetivas, ou seja, as emoções, considerando que o organismo possui uma certa dificuldade em lidar com as emoções, traduzindo-as em doenças por sentir-se ameaçado e em risco.

Segundo Jorge (1995), ao se conhecer as causas e motivos que desencadeiam o ato da falta, pode-se colaborar com o processo de humanização dos hospitais, 
facilitando as escalas de trabalho, para tentar diminuir os dias duvidosos por dias de efetivo trabalho e para aumentar o grau de satisfação no trabalho, tentando com esta estratégia, diminuir o número de ausências, conciliando assim, os interesses do funcionário com os institucionais.

No entanto, os estudos realizados por Gaidzinski et al (1993), Gaidzinski et al (1998) têm demonstrado que são as ausências previstas (folgas e férias), as que mais interferem no quantitativo de pessoal de enfermagem que efetivamente está trabalhando, uma vez que esses índices são elevados, principalmente no que se refere as ausências por folgas. Como na maioria dos hospitais não há cobertura para os dias de folga, o quadro de pessoal, que via de regra já é subestimado, fica extremamente prejudicado, causando sobrecarga de trabalho para equipe de enfermagem, o que pode comprometer a qualidade da assistência prestada à clientela.

Acreditamos que frente a essa situação, o absenteísmo (ausências não previstas) acabe por chamar tanta atenção dos administradores da instituição, uma vez que pode dar a impressão que a falta de funcionários seja decorrente do número excessivo de faltas e licenças. No entanto, na maioria das vezes isso não corresponde a realidade.

Os estudos sobre as ausências têm sido, praticamente, realizados apenas em instituições hospitalares que trabalham em um regime de trabalho de 36h/ semanais, com jornada de 6h/dia (Gaidzinski et al, 1993, Gaidzinski et al, 1998, Fugulin, 1998).

Diante disso, resolvemos nesse estudo investigar, em uma instituição hospitalar, como as ausências previstas e não previstas se configuram em um regime de trabalho de trabalho de 40h/semanais, com jornada de 8h/dia.

\section{OBJETIVOS}

Essa pesquisa tem por objetivos:

caracterizar e analisar os índices de ausências previstas e não previstas,da equipe de enfermagem, em uma instituição de saúde com carga horária semanal de 40 horas e jornada diária de 8 horas;

comparar os índices encontrados, nesse regime de trabalho, entre as unidades da instituição e com os indicados na literatura.

\section{MATERIAL E MÉTODO}

Trata-se de estudo exploratório realizado em uma instituição hospitalar, onde, inicialmente foram feitos contatos, com a diretoria de enfermagem e com o responsável pelo departamento de pessoal, a fim de solicitar autorização e colaboração para o desenvolvimento dessa investigação.

\section{LOCAL DO ESTUDO}

A pesquisa foi realizada em um hospital geral de grande porte, pertencente ao município de São Paulo, que atende quatro especialidades básicas: pediatria, obstetrícia e ginecologia, cirurgia geral e clínica médica.

Nesse hospital são atendidos pacientes através do Sistema Único de Saúde (SUS), particulares, conveniados com alguns planos de saúde e sócios da instituição. 
Assim, cerca de $50 \%$ são pacientes provenientes do SUS, $40 \%$ são conveniados, $10 \%$ particulares e associados da instituição.

Esse local foi selecionado para o estudo, pela falta de conhecimento de como se caracteriza a distribuição das ausências da equipe de enfermagem trabalhando, em um regime de 40 horas semanais, com jornada de 08 horas diárias.

\section{POPULAÇÃO E AMOSTRA}

A população estudada foi constituída pelos enfermeiros, auxiliares e atendentes de enfermagem que integram a equipe de enfermagem das Unidades de Internação, do Centro Cirúrgico, da UTI Geral e Pediátrica.

A amostragem foi definida a partir do número total desses funcionários de enfermagem distribuídos nessas unidades, nos três turno, no período de janeiro a dezembro de 1996.

Os dados referentes a distribuição dos funcionários nas unidades foi obtido através de uma listagem dividida por centros de custos em: centro de custo referente à UTI Geral, UTI Pediátrica, Unidades de Internação e Centro Cirúrgico, pois esses centros de custos representavam, significativamente, o total do número de funcionários da enfermagem do hospital. Foram excluídos da amostra, os funcionários que foram admitidos ou demitidos no transcorrer do ano de 1996.

No centro de custo referente à UTI Geral, temos 165 leitos no total, distribuídos em 18 leitos de cirurgia pediátrica cardiológica, 16 leitos para neurologia, 22 leitos para pacientes gerais e 109 leitos para pacientes cirúrgicos cardiológicos. Nesse centro de custo encontramos 326 funcionários de enfermagem, sendo 265 auxiliares de enfermagem, 30 enfermeiros, 31 atendentes. Para determinação da amostra partimos do número total de enfermeiros, por ser o menor grupo de profissionais dessa área, definindo-se a amostra em: 30 enfermeiros; 60 auxiliares e 31 atendentes de enfermagem.

Na UTI Pediátrica temos 08 leitos e 27 funcionários no total, que dividem-se em 04 enfermeiras, 20 auxiliares, 03 atendentes, devido ao número de funcionários, toda a população foi estudada.

O Centro Cirúrgico possui 38 salas de cirurgia e 123 funcionários de enfermagem no total, divididos em 08 enfermeiras, 63 auxiliares, 52 atendentes. Assim a amostra constitui-se de: 07 enfermeiros, 51 auxiliares e 42 atendentes.

O Centro de Custo Enfermagem corresponde às Unidades de Internação, perfazendo 1052 leitos e 539 funcionários de enfermagem no total, sendo 42 enfermeiros, 396 auxiliares e 101 atendentes. A amostra ficou definida em: 41 enfermeiros, 80 auxiliares e 53 atendentes.

\section{PROCEDIMENTO PARA COLETA DOS DADOS}

Os dados referentes aos dias de ausências dos funcionários foram coletados através das fichas de freqüência de controle do hospital. Essas fichas contêm os dados referentes aos dias de ausência devido a licença: maternidade, acidente de trabalho, médica; férias; faltas injustificadas e justificadas e suspensão. O registro dessas ausências é feito mensalmente, pelo departamento de pessoal nessa ficha 
para cada funcionário.

As fichas foram escolhidas aleatoriamente, segundo o número da amostra de cada elemento da equipe das unidades escolhidas nesse estudo, não sendo considerado o turno de trabalho. Esses dados foram registrados em número de dias de cada tipo de ausência, ocorridas durante o ano de 1996 e organizados em um instrumento tipo planilha. Recomenda-se o período de um ano para o estudo das ausências para que possa-se analisar os índices de todos os tipos de ausências que ocorrem no decorrer de um ano.

As ausências por folgas foram calculadas a partir do número de finais semana do ano, pois nesse regime cada funcionário possui duas folgas semanais, o que representa 104 dias de folgas por funcionário e acrescidas por mais 8 dias de folgas devido aos dias feriados do ano que não coincidiram com os finais de semana, obtendo-se assim um número de 9,3 dias de folgas mensais por funcionário.

Para o cálculo percentual de cada tipo de ausência adotou-se a equação proposta por Gaidzinski (1991):

$A \%=\frac{N .100}{F . D}$

onde:

$A \%=$ percentual de ausências;

$\mathrm{N}=$ número dias de ausências de janeiro a dezembro de 1996;

$\mathrm{F}=$ número funcionários

$D$ = dias possíveis de ocorrer a ausência, no período de um ano assim temos:

D para folgas (descanso remunerado semanal e feriados):

$\mathrm{D}=365-\frac{\mathrm{N}_{\text {(folgas) }}}{\mathrm{F}}$

$D$ para férias:

$D=365-\frac{N_{\text {(folgas) }}}{F}$

D para ausências não previstas

$\mathrm{D}=365-\frac{N_{\text {(folgas) }}}{\mathrm{F}}-\frac{N_{\text {(folgas) }}}{\mathrm{F}}$

As faltas justificadas, injustificadas e abonadas foram todas agrupadas. Como outras licenças consideram-se os seguintes tipos: nojo, gala e paternidade.

\section{APRESENTAÇÃO E ANÁLISE DOS DADOS}

A pesquisa englobou a análise das ausências previstas (folgas e férias) e das ausências não previstas (faltas, licenças e suspensões) de 421 elementos da equipe de enfermagem, destes 370 (88\%) são do sexo feminino e 51 (12\%) são de sexo masculino.

A análise dos dados deu-se em função do percentual expresso nos dados 
apresentados a seguir de ausências previstas e não previstas da equipe de enfermagem por unidade estudada:

Os dados estão apresentados e tabulados nos Quadros 1, 2, 3 e 4.

Como resultado geral, verificamos que o índice de ausências previstas de todas as unidades é significativamente maior que o índice de ausências não previstas. As ausências previstas perfazem quase a totalidade das ausências,

QUADRO 1: Demonstrativo das ausências previstas e não previstas da equipe de enfermagem na Unidade de Centro Cirúrgico, São Paulo, 1996.

\begin{tabular}{|c|c|c|c|c|c|c|c|c|c|c|c|c|}
\hline \multirow{2}{*}{$\begin{array}{l}\text { CATEGORIA } \\
\text { PROFISSIONAL } \\
\text { OU FUNÇĀO }\end{array}$} & \multirow{2}{*}{$\begin{array}{l}\text { QUANT. } \\
\text { MEDIA DE } \\
\text { PESSOAL }\end{array}$} & \multicolumn{3}{|c|}{ AUSÊNCIAS PREVISTAS } & \multicolumn{7}{|c|}{ AUSÊNCIAS NĀO PREVISTAS } & \multirow{2}{*}{$\begin{array}{l}\text { T } \\
O \\
T \\
A \\
\text { L }\end{array}$} \\
\hline & & FOLGAS & FÉRIAS & $\begin{array}{l}\text { SUB } \\
\text { TOTAL }\end{array}$ & FALTAS & $\begin{array}{l}\text { LICENÇA } \\
\text { MÉDICA }\end{array}$ & $\begin{array}{l}\text { LICENÇA } \\
\text { MATERN. }\end{array}$ & $\begin{array}{l}\text { LICENÇA } \\
\text { ACIDENTE }\end{array}$ & $\begin{array}{l}\text { OUTRAS } \\
\text { LICENÇAS }\end{array}$ & $\begin{array}{l}\text { SUSPEN- } \\
\text { SŌES }\end{array}$ & $\begin{array}{l}\text { SUB } \\
\text { TOTAL }\end{array}$ & \\
\hline \multirow[t]{2}{*}{ ENFERMEIRO } & \multirow[t]{2}{*}{7.00} & 784 & 179 & 963 & 4 & 2 & 0 & 0 & 0 & 0 & 23 & 903 \\
\hline & & $44,27 \%$ & $7,53 \%$ & $51,80 \%$ & $016 \%$ & $0,08 \%$ & $0,00 \%$ & $0,00 \%$ & $0.00 \%$ & $0,00 \%$ & $1.60 \%$ & $52.59 \%$ \\
\hline \multirow[t]{2}{*}{ AUXILIAR } & \multirow[t]{2}{*}{51.00} & 5712 & 1382 & 7094 & 126 & 192 & 120 & 23 & 0 & 4 & 146 & 2881 \\
\hline & & $44,27 \%$ & $8,02 \%$ & $52,29 \%$ & $0,69 \%$ & $1,06 \%$ & $0,66 \%$ & $0.13 \%$ & $0,00 \%$ & $0,06 \%$ & $2,04 \%$ & $53,58 \%$ \\
\hline \multirow[t]{2}{*}{ ATENDENTE } & \multirow[t]{2}{*}{42.00} & 4704 & 1159 & 5863 & 132 & 303 & 168 & 0 & 0 & 3 & 28 & 392 \\
\hline & & $44,27 \%$ & $8,18 \%$ & $52,45 \%$ & $0,90 \%$ & $2,06 \%$ & $1,14 \%$ & $0,00 \%$ & $0.00 \%$ & $0,28 \%$ & $2,62 \%$ & $49.52 \%$ \\
\hline \multirow[t]{2}{*}{ TOTAL } & 100,00 & 11200 & 2720 & 13920 & 262 & 497 & 288 & 23 & 0 & 7 & 197 & 3836 \\
\hline & & $44.27 \%$ & $8,05 \%$ & $52.32 \%$ & $0,74 \%$ & $1,40 \%$ & $0.81 \%$ & $0.06 \%$ & $0.00 \%$ & $0.07 \%$ & $2.04 \%$ & $52.96 \%$ \\
\hline
\end{tabular}

QUADRO 2: Demonstrativo das ausências previstas e não previstas da equipe de enfermagem na Unidade de Internação, São Paulo, 1996.

\begin{tabular}{|c|c|c|c|c|c|c|c|c|c|c|c|c|}
\hline \multirow{2}{*}{$\begin{array}{l}\text { CATEGORIA } \\
\text { PROFISSIONAL } \\
\text { OU FUNÇĀO }\end{array}$} & \multirow{2}{*}{$\begin{array}{l}\text { QUANT. } \\
\text { MÉDIA DE } \\
\text { PESSOAL }\end{array}$} & \multicolumn{3}{|c|}{ AUSÊNCIAS PREVISTAS } & \multicolumn{7}{|c|}{ AUSÊNCIAS NĀO PREVISTAS } & \multirow{2}{*}{$\begin{array}{l}\mathrm{T} \\
\mathrm{O} \\
\mathrm{T} \\
\mathrm{A} \\
\mathrm{L}\end{array}$} \\
\hline & & FOLGAS & FÉRIAS & $\begin{array}{l}\text { SUB } \\
\text { TOTAL }\end{array}$ & FALTAS & $\begin{array}{l}\text { LICENÇA } \\
\text { MÉDICA }\end{array}$ & $\begin{array}{l}\text { LICENÇA } \\
\text { MATERN. }\end{array}$ & $\begin{array}{l}\text { LICENÇA } \\
\text { ACIDENTE }\end{array}$ & $\begin{array}{l}\text { OUTRAS } \\
\text { LICENÇAS }\end{array}$ & $\begin{array}{l}\text { SUSPEN- } \\
\text { SÖES }\end{array}$ & $\begin{array}{c}\text { SUB } \\
\text { TOTAL }\end{array}$ & \\
\hline \multirow[t]{2}{*}{ ENFERMEIRO } & \multirow[t]{2}{*}{41,00} & 4208 & 1164 & 5372 & 44 & 150 & 120 & 8 & 0 & 5 & 327 & 5699 \\
\hline & & $40,57 \%$ & $8,43 \%$ & $49,00 \%$ & $0.46 \%$ & $1,56 \%$ & $1,25 \%$ & $0,08 \%$ & $0.00 \%$ & $0,05 \%$ & $3.41 \%$ & $52,41 \%$ \\
\hline \multirow[t]{2}{*}{ AUXILIAR } & \multirow[t]{2}{*}{80,00} & 8210 & 2274 & 10484 & 225 & 174 & 120 & 0 & 0 & 17 & 536 & 11020 \\
\hline & & $40,56 \%$ & $8.45 \%$ & $49,01 \%$ & $1,20 \%$ & $0,93 \%$ & $0,64 \%$ & $0,00 \%$ & $0,00 \%$ & $0,09 \%$ & $2.86 \%$ & $51,87 \%$ \\
\hline \multirow[t]{2}{*}{ ATENDENTE } & \multirow[t]{2}{*}{53.00} & 5439 & 1146 & 6585 & 157 & 303 & 0 & 9 & 0 & 14 & 483 & 7068 \\
\hline & & $40,56 \%$ & $6,30 \%$ & $46,86 \%$ & $1,23 \%$ & $2,37 \%$ & $0,00 \%$ & $0,07 \%$ & $0,00 \%$ & $0,11 \%$ & $3,79 \%$ & $50,64 \%$ \\
\hline \multirow[t]{2}{*}{ TOTAL } & 174.00 & 17857 & 4584 & 22441 & 426 & 627 & 240 & 17 & 0 & 36 & 1346 & 23787 \\
\hline & & $40,56 \%$ & $7.78 \%$ & $48,34 \%$ & $1.04 \%$ & $1,53 \%$ & $0.58 \%$ & $0,04 \%$ & $0,00 \%$ & $0.09 \%$ & $3.28 \%$ & $51,62 \%$ \\
\hline
\end{tabular}


QUADRO 3: Demonstrativo das ausências previstas e não previstas da equipe de enfermagem na Unidade de Terapia Intensiva, São Paulo, 1996.

\begin{tabular}{|c|c|c|c|c|c|c|c|c|c|c|c|c|}
\hline \multirow{2}{*}{$\begin{array}{l}\text { CATEGORIA } \\
\text { PROFISSIONAL } \\
\text { OU FUNÇĀO }\end{array}$} & \multirow{2}{*}{$\begin{array}{l}\text { QUANT. } \\
\text { MÉDIA DE } \\
\text { PESSOAL }\end{array}$} & \multicolumn{3}{|c|}{ AUSEENCIAS PREVISTAS } & \multicolumn{7}{|c|}{ AUSÊNCIAS NĀO PREVISTAS } & \multirow{2}{*}{$\begin{array}{l}\text { T } \\
\text { O } \\
\text { T } \\
\text { A } \\
\text { L }\end{array}$} \\
\hline & & FOLGAS & FÉRIAS & $\begin{array}{l}\text { SUB } \\
\text { TOTAL }\end{array}$ & FALTAS & $\begin{array}{l}\text { LICENÇA } \\
\text { MÉDICA }\end{array}$ & $\begin{array}{l}\text { LICENÇA } \\
\text { MATERN. }\end{array}$ & $\begin{array}{l}\text { LICENÇA } \\
\text { ACIDENTE }\end{array}$ & $\begin{array}{l}\text { OUTRAS } \\
\text { LICENÇAS }\end{array}$ & $\begin{array}{l}\text { SUSPEN- } \\
\text { SŌES }\end{array}$ & $\begin{array}{l}\text { SUB } \\
\text { TOTAL }\end{array}$ & \\
\hline \multirow[t]{2}{*}{ ENFERMEIRO } & \multirow[t]{2}{*}{30,00} & 3079 & 894 & 3973 & 15 & 59 & 250 & 0 & 0 & 0 & 324 & 4297 \\
\hline & & $40,57 \%$ & $8,89 \%$ & $49,46 \%$ & $0,21 \%$ & $0,85 \%$ & $3,58 \%$ & $0,00 \%$ & $0,00 \%$ & $0,00 \%$ & $4.64 \%$ & $54.10 \%$ \\
\hline \multirow[t]{2}{*}{ AUXILIAR } & \multirow[t]{2}{*}{60.00} & 6158 & 1659 & 7817 & 155 & 216 & 337 & 86 & 0 & 21 & 815 & 8632 \\
\hline & & $40.57 \%$ & $8,20 \%$ & $48,76 \%$ & $1.10 \%$ & $1,53 \%$ & $2,39 \%$ & $0,61 \%$ & $0,00 \%$ & $0,15 \%$ & $5.79 \%$ & $54,55 \%$ \\
\hline \multirow[t]{2}{*}{ ATENDENTE } & \multirow[t]{2}{*}{30,00} & 3079 & 1121 & 4200 & 70 & 78 & 507 & 1 & 0 & 7 & 663 & 4863 \\
\hline & & $40.57 \%$ & $11,41 \%$ & $51,97 \%$ & $1,04 \%$ & $1,16 \%$ & $7.51 \%$ & $0,01 \%$ & $0,00 \%$ & $0,10 \%$ & $9,82 \%$ & $61,79 \%$ \\
\hline \multirow[t]{2}{*}{ TOTAL } & 120,00 & 12316 & 3674 & 15990 & 240 & 353 & 1094 & 87 & 0 & 28 & 1802 & 17792 \\
\hline & & $40.57 \%$ & $9,16 \%$ & $49,72 \%$ & $0,86 \%$ & $1.27 \%$ & $3,93 \%$ & $0.31 \%$ & $0,00 \%$ & $0.10 \%$ & $6,48 \%$ & $56,20 \%$ \\
\hline
\end{tabular}

QUADRO 4: Demonstrativo das ausências previstas e não previstas da equipe de enfermagem na Unidade de Terapia Intensiva Pediátrica, São Paulo, 1996.

\begin{tabular}{|c|c|c|c|c|c|c|c|c|c|c|c|c|}
\hline \multirow{2}{*}{$\begin{array}{l}\text { CATEGORIA } \\
\text { PROFISSIONAL } \\
\text { OU FUNÇĀO }\end{array}$} & \multirow{2}{*}{$\begin{array}{l}\text { QUANT. } \\
\text { MÉDIA DE } \\
\text { PESSOAL }\end{array}$} & \multicolumn{3}{|c|}{ AUSÊNCIAS PREVISTAS } & \multicolumn{7}{|c|}{ AUSÉNCIAS NĀO PREVISTAS } & \multirow{2}{*}{$\begin{array}{l}\mathrm{T} \\
\mathrm{O} \\
\mathrm{T} \\
\mathrm{A} \\
\mathrm{L}\end{array}$} \\
\hline & & FOLGAS & FÉRIAS & $\begin{array}{l}\text { SUB } \\
\text { TOTAL }\end{array}$ & FALTAS & $\begin{array}{l}\text { LICENÇA } \\
\text { MÉDICA }\end{array}$ & $\begin{array}{l}\text { LICENÇA } \\
\text { MATERN. }\end{array}$ & $\begin{array}{l}\text { LICENÇA } \\
\text { ACIDENTE }\end{array}$ & $\begin{array}{l}\text { OUTRAS } \\
\text { LICENÇAS }\end{array}$ & $\begin{array}{l}\text { SUSPEN- } \\
\text { SÖES }\end{array}$ & $\begin{array}{l}\text { SUB } \\
\text { TOTAL }\end{array}$ & \\
\hline \multirow[t]{2}{*}{ ENFERMEIRO } & \multirow[t]{2}{*}{4,00} & 410 & 92 & 502 & 7 & 16 & 0 & 0 & 0 & 0 & 23 & 525 \\
\hline & & $40,51 \%$ & $6,73 \%$ & $47.24 \%$ & $0,73 \%$ & $1,67 \%$ & $0,00 \%$ & $0,00 \%$ & $0,00 \%$ & $0.00 \%$ & $2.40 \%$ & $49,64 \%$ \\
\hline \multirow[t]{2}{*}{ AUXILIAR } & \multirow[t]{2}{*}{20.00} & 2052 & 495 & 2547 & 72 & 70 & 0 & 0 & 0 & 4 & 146 & 2693 \\
\hline & & $40,55 \%$ & $7,27 \%$ & $47,83 \%$ & $1,51 \%$ & $1,47 \%$ & $0,00 \%$ & $0,00 \%$ & $0,00 \%$ & $0,08 \%$ & $3.07 \%$ & $50,90 \%$ \\
\hline \multirow[t]{2}{*}{ ATENDENTE } & \multirow[t]{2}{*}{3.00} & 308 & 28 & 336 & 25 & 0 & 요 $10 \mathrm{rar}-\mathrm{r}$ & 0 & 0 & 3 & 28 & 364 \\
\hline & & $40,58 \%$ & $2,62 \%$ & $43,20 \%$ & $3.29 \%$ & $0,00 \%$ & $0,00 \%$ & $0,00 \%$ & $0,00 \%$ & $0.40 \%$ & $3,69 \%$ & $46,89 \%$ \\
\hline \multirow[t]{2}{*}{ TOTAL } & 27,00 & 2770 & 615 & 3385 & 104 & 86 & 0 & 0 & 0 & 7 & 197 & 3582 \\
\hline & & $40,55 \%$ & $6,66 \%$ & $47,21 \%$ & $1,61 \%$ & $1,33 \%$ & $0,00 \%$ & $0,00 \%$ & $0,00 \%$ & $0.11 \%$ & $3,04 \%$ & $50,25 \%$ \\
\hline
\end{tabular}

sendo que o tipo de ausência prevista mais expressivo refere-se às folgas, $(40,56 \%)$ uma vez que essa instituição oferece duas folgas semanais em virtude da carga horária ser de 40h, numa jornada de 8 h diárias.

Observa-se também que os percentuais correspondentes aos dias de férias é o segundo maior percentual de ausências, representando, praticamente, 9,6\%. 
GRÁFICO 1 - Índice geral das ausências previstas e não previstas da equipe de enfermagem no período de jan / dez - 1996.

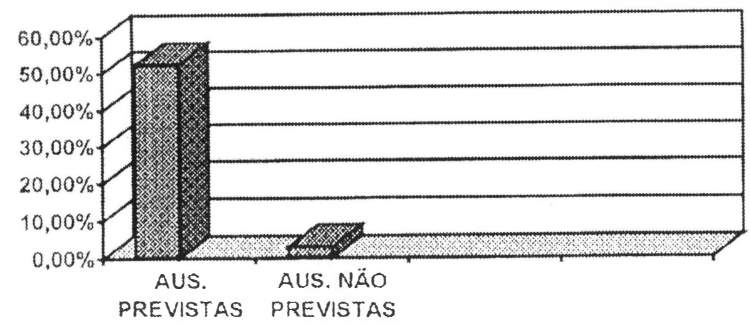

Cumpre lembrar, que essas ausências são de direito de todos trabalhadores da enfermagem e correspondem, praticamente a 50\% de pessoas ausentes ao trabalho, por folgas e férias. Ou seja, há necessidade de previsão de $50 \%$ a mais do quadro pessoal de enfermagem previsto, para que se tenha a cobertura para esses tipos de ausência.

No estudo de Alcalá et al (1982) a taxa de ausências previstas citada é de 24\%. Baptista (1976) encontrou um índice de ausências previstas de 30\%.

A recomendação atual proposta pela Resolução COFEN no 189/96, também não se aplica ao local estudado, pois a defasagem de pessoal por ausências previstas é maior do que 30\%. Salientamos portanto a importância de se realizarem estudos quantitativos para determinar a necessidade de cobertura de pessoal para ausências previstas nas instituições hospitalares, uma vez que o quantitativo de folgas varia de acordo com a carga horária semanal e com a jornada de trabalho.

No que se refere às ausências não previstas: faltas, licenças e suspensões como mostra o Quadro 5, o índice foi baixo, em média 3\%. Assim, esse grupo de funcionários apresenta uma taxa de absenteísmo reduzida em relação aos dados da literatura.

Em relação a jornada de trabalho, Gillespi (1996) comparando turnos de 12 horas e turnos de 8 horas verificou que não houve alteração significativa em relação ao número de ausências dos funcionários. Baptista (1976) também comparou turnos de 44 e 40 horas semanais, e concluiu que não houveram mudanças estatisticamente significativas em relação ao número de ausências não previstas.

De acordo com Kerper (1971), a taxa geral de absenteísmo nos hospitais americanos é de 4\%. No Brasil, Baptista (1976) ao estudar a taxa de absenteísmo em um hospital de ensino identificou que esta taxa variou de $16 \%$ no máximo e 3\% no mínimo. Assim podemos considerar que comparado aos índices encontrados na literatura pertinente, o índice de ausências não previstas na instituição em estudo, foi baixo.

Ao analisarmos as ausências não previstas por categoria (Quadro 5), verificamos que os atendentes de enfermagem apresentaram os maiores índices de absenteísmo, com uma média de $4 \%$ de ausências não previstas. Baptista (1976) também encontrou os maiores índices de ausências não previstas na categoria atendente de enfermagem. 
Isso sugere que as condições de vida ou de trabalho precisam ser consideradas como um fator importante ao se relacionar as causas das ausências dos funcionários. Outro fator a ser considerado é o sexo dos funcionários pois a amostra para esse estudo foi composta na sua maioria, por mulheres. Assim, a licença maternidade em algumas unidades foi um índice significativo dentro das ausências não previstas.

Ao compararmos as ausências não previstas segundo o tipo de unidade percebemos que o maior índice recai sobre a Unidade de Terapia Intensiva (Gráfico 2).

GRÁFICO 2 - Índice das ausências não previstas da equipe de enfermagem, segundo a unidade estudada, no período de jan / dez - 1996.

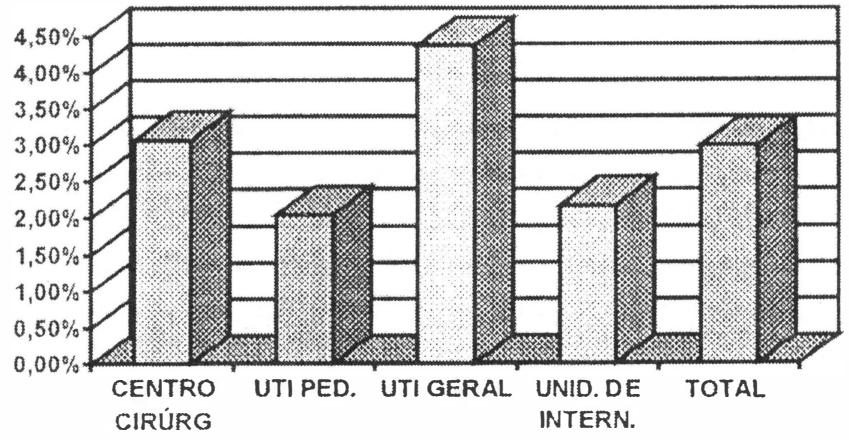

No entanto, ao analisarmos o Quadro 3 verificamos que esse índice foi mais elevado devido à licença maternidade.

\section{CONCLUSÕES}

Os resultados dessa investigação levaram às seguintes conclusões:

- O índice médio de ausências previstas (52,53\%) foi significativamente maior que o índice de ausências não previstas (4\%), demonstrando que as ausências dos funcionários dessa instituição ocorreram por folgas e férias, ausências estas que constituem-se direito do trabalhador.

- O índice mais expressivo refere-se as folgas pois esse regime de trabalho oferece duas folgas semanais por funcionário.

- As ausências previstas são as responsáveis por cerca de 50\% de pessoas ausentes nas unidades, havendo portanto, a necessidade de cobertura dessas ausências.

O índice de ausências não previstas e que caracteriza o absenteísmo corresponde a 3\%. Esse valor é considerado baixo em relação aos dados da literatura. 


\section{REFERÊNCIAS BIBLIOGRÁFICAS}

1. ALCALÁ, M.U.; et al. Cálculo de pessoal: estudo preliminar para estabelecimento de quadro de pessoal de enfermagem na superintendência médico hospitalar de urgência. São Paulo. Secretaria de Higiene e Saúde, 1982.

2. ALVES, M. As causas do absenteísmo na enfermagem: uma visão do sofrimento no trabalho. São Paulo, 1996. 142p. Tese (Doutorado) - Escola de Enfermagem da Universidade de São Paulo.

3. BAPTISTA, W.A. Frequência de absentismo de pessoal de enfermagem em um hospital de ensino. Rev. Paul. Hosp. V.24, n.11, 1976.

4. ChIAVEnAtO, I. Recursos humanos na empresa. São Paulo, Atlas, 1991. P.119-20.

5. CONSELHO FEDERAL DE ENFERMAGEM. Resolução no 189/96. Estabelece parâmetros para dimensionamento do quadro de profissionais de enfermagem nas instituições de saúde. In.: CONSELHO REGIONAL DE ENFERMAGEM. Documentos básicos de enfermagem: enfermeiros, técnicos e auxiliares. São Paulo, 1997. p.177-80.

6. COUTO, H.A. Absenteísmo: uma visão bem maior que a simples doença. In.: COUTO, H.A. Temas de saúde ocupacional. Belo Horizonte, Ergos, p.9-37, 1987.

7. DIAS, M.A.deA.; AVELAR, M.do C.Q. Absenteísmo em enfermagem: o enfoque da sua redução. / Apresentado no $48^{\circ}$ Congresso Brasileiro de Enfermagem, São Paulo, 1996.

8. FELT, B.L. Absenteeism in nursing. Nurs. Manag. 1982, v.13, n.1, p.35-8. 1982.

9. FLIPPO, E.B. Princípios de Administração de Pessoal. São Paulo, Atlas, 1970.

10.FRENKIEL, S. O absenteísmo em empresa ferroviária. IN.: CONGRESSO NACIONAL DE PREVENÇÃO DE ACIDENTES DE TRABALHO, Brasília, Fundacentro, 1980. Anais. P.420-34. 1980.

11.FUGULIN, F.M.T. Sistema de classificação de pacientes: análise das horas de assistência de enfermagem. São Paulo, 1997. 75p. Dissertação (Mestrado) - Escola de Enfermagem, Universidade de São Paulo.

12.GAIDZINSKI, R.R. ; et al. Estudo das ausências da equipe de enfermagem num hospital geral de grande porte. Rev. Enf. Complexo HC/FMUSP, V.1, N.4, P.8-14, 1998. 
13. GAIDZINSKI, R.R.; et al. O estudo das ausências da equipe de enfermagem. / Apresentado ao $2^{\circ}$ Encontro de Enfermeiros de Hospitais de Ensino do Estado de São Paulo, Campinas, 1993/

14. GILLESPIE, A. A comparison of a 12 hour and 8 hour shift system. Nursing Times, v.96, n.39, p.36-39, 1996.

15. JORGE, A.L.S. Motivos que levamos trabalhadores de enfermagem ao absenteísmo. Acta Paul. Enf., São Paulo, v.8, n., p.39-46, 1995.

16. KERPER, R.E.Jr. Toward a solution to abseteeism. Occup. Health. Nurs. V.19, n.12, p.7-8,41, 1971.

17. LOMAN, D.G. Child care problems of nurses. J.Nurs. Adm., v.23, n.3, p.5-6, 1993.

18. ORGANIZACIÓN INTERNACIONAL DEL TRABAJO. Absentismo, causas y control. In.: Enciclopedia de salud y seguridad en el trabajo. Madri, v.1, p.5-12, 1989.

19. PLATI; et al. Nursing absenteeism - one determining factor for the staff plan. Scand. J. Caring. Sci. V.8, n.3, 1990.

20. ROBAZZI, M.L.C.C.; etal. Serviço de enfermagem: um estudo sobre absenteísmos. Rev. Bras. Saúde Ocup., v.18, n.69, p.65-70, 1990.

21. ROGERS, J.E.; HUTCHINS, S.G.; JOHNSON, B.J. Nonpunitive discipline: a method of reducing absenteeism. JONA, v.20, n.7/8, p.41-3, 1990.

22. SILVA, M.da. A. A produção científica sobre a relação trabalho e saúde na enfermagem: questões para debate. Rev. Enf. UERJ, v.4, n.1, 27-38, 1996.

23. STEAGAL-GOMES, D.L. : MENDES, J.J.M. A força de trabalho da mulher. Acta.Paul. Enf. São Paulo, v.8, n.1, p.39-46, 1995.

24. SULLIVAN, E.J.; DECKER, P.J. Effective management in nursing. Redwood City, CA, Addidson-Wesley, 1992. 